\title{
Holographic pseudo-Goldstone boson
}

\author{
Adam Falkowski* \\ CERN Theory Division, CH-1211 Geneva 23, Switzerland \\ and Institute of Theoretical Physics, Warsaw University, Hoza 69, 00-681 Warsaw, Poland
}

(Received 3 November 2006; published 19 January 2007)

\begin{abstract}
Pseudo-Goldstone bosons in 4D strongly coupled theories have a dual description in terms of 5D gauge theories in warped backgrounds. We introduce systematic methods of computing the pseudo-Goldstone potential for an arbitrary warp factor in 5D. When applied to electroweak symmetry breaking, our approach clarifies the relation of physical observables to geometrical quantities in five dimensions.
\end{abstract}

PACS numbers: 12.10.Dm

\section{INTRODUCTION}

The LHC experiment will soon probe how $W$ and $Z$ bosons acquire their masses. So far we have only indirect hints about the actual mechanism of electroweak symmetry breaking. The standard model (SM) introduces a weakly coupled fundamental scalar field-the Higgs fieldwhose condensation breaks the electroweak symmetry. This simple picture is consistent with precision electroweak measurements, if the mass of the Higgs boson is $\gtrsim 100 \mathrm{GeV}$ and the SM cutoff is far above a TeV. However, from a theoretical point of view, a fundamental scalar much lighter than the theory's cutoff is unnatural, unless there is a symmetry protecting its mass.

Perhaps the most elegant realization of the Higgs sector is the one in which the Higgs field is composite, rather than fundamental [1]. This can be achieved by introducing a new strongly interacting sector with a global symmetry $G \supset G_{\mathrm{SM}}$. In this scenario the Higgs field is a pseudoGoldstone boson arising from a spontaneous breaking of $G$. The global symmetry could be broken by a condensate, in close analogy with chiral symmetry breaking in QCD. If the condensate leaves the SM gauge symmetry intact, the effective theory below the compositeness scale of the new strong interactions includes the SM fields coupled to a nonlinear sigma model field describing the pseudoGoldstone Higgs. The Higgs field has a flat potential at tree level, but a nontrivial potential can be generated through its couplings to the SM that do not respect the full global symmetry. In such framework, we expect the Higgs potential of the form

$$
V(h) \sim \frac{1}{4 \pi^{2}} \int_{0}^{\infty} d p p^{3} \log \left(1+F\left(p^{2}\right) \sin ^{2}\left(h / f_{h}\right)\right) .
$$

Here $f_{h}$ is the scale of the spontaneous global symmetry breaking. The potential depends on the Higgs via $\sin \left(h / f_{h}\right)$ because Goldstone fields are described by periodic variables. The form factor $F\left(p^{2}\right)$ depends on the effective theory interactions between the SM gauge and fermion fields and the pseudo-Goldstone Higgs.

\footnotetext{
*Email:adam.falkowski@cern.ch
}

The main drawback of this scenario is that strongly coupled theories are notoriously difficult to master. Typically, the form factors can only be estimated by naive dimensional analysis. However, it has recently been realized that at least some 4D strongly coupled sectors - those with a large number of colors of the strong gauge groupcan be represented by perturbative 5D gauge theories. The $5 \mathrm{D}$ gravitational background is curved with the line element $d s^{2}=a^{2}\left(x_{5}\right)(d x)^{2}-d x_{5}^{2}$ and the 5th dimension is truncated by a UV brane at $x_{5}=0$ and by an IR brane at $x_{5}=L$. This is an example of holography [2] applied to 5D field theories [3]. According to the holographic dictionary, the 5D gauge symmetry $G$ broken by boundary conditions on the IR brane corresponds to the spontaneously broken global symmetry $G$. Perturbative expansion in the 5D gauge coupling $g_{5}$ corresponds to $1 / N_{c}$ expansion in 4D. On the 5D side, the spectrum contains a scalar excitation originating from the 5th component of the gauge field along the broken gauge group generators. This excitation can be identified with the 4D pseudo-Goldstone boson. Next, the 5D gauge symmetry is reduced on the UV brane, which corresponds to explicit breaking of the global symmetry in $4 \mathrm{D}$ by couplings of external fields to the strongly interacting sector. The explicit breaking generates a potential for the pseudo-Goldstone Higgs. The important advantage of the 5D approach is that the Higgs potential can be computed perturbatively.

In fact, computations of the pseudo-Goldstone Higgs potential in the $5 \mathrm{D}$ framework were first performed independently of the holographic interpretation [4]. Instead, the motivation was the identification of the Higgs field with a component of a gauge field. This gauge-Higgs unification provides a solution to the hierarchy problem from the 5D perspective. Indeed, loop corrections to the Higgs potential are necessarily finite; the divergent corrections are not allowed because the gauge-invariant operator that can yield a Higgs mass is nonlocal in the 5 th coordinate.

At the phenomenological level, most of the activity so far has concentrated on gauge-Higgs unification in the flat Minkowski background [5], which does not admit a holographic interpretation. Generalization to the $\mathrm{AdS}_{5}$ background (which corresponds to conformal symmetry of the 
strongly coupled sector) was accomplished quite recently, in [6-10] and the holographic aspect was stressed in $[6,8]$. The models in $\mathrm{AdS}_{5}$ have reached the stage of fully realistic extensions of the SM, as shown by the calculation of electroweak observables in [11].

In this paper we advocate methods that allow the computation of the form factors entering the pseudo-Goldstone potential for an arbitrary 5D warped background. This generalization corresponds to deviations of the 4D strong sector from conformal symmetry. Such deviations are, in fact, expected in realistic models of the strong sector, in which the hierarchy of scales between the UV brane and the IR brane is stabilized [12]. We show that the form factors can be simply expressed in terms of the solutions to the 5D equations of motion. Moreover, in many cases the form factors can be adequately approximated by a simple analytic expression:

$$
F\left(-p^{2}\right) \approx \frac{y^{2} f_{h}^{2}}{M_{\mathrm{KK}}^{2}} \frac{1}{\sinh ^{2}\left(p / M_{\mathrm{KK}}\right)},
$$

where $y$ stands for a low-energy Yukawa or gauge coupling. The Kaluza-Klein (KK) scale $M_{\mathrm{KK}}$ is roughly equal the mass of the lightest $\mathrm{KK}$ mode and corresponds to the compositeness scale in 4D. At low energies, below the KK/ compositeness scale, the form factor exhibits the $1 / p^{2}$ behavior characteristic of pointlike particles. The 5D framework predicts that above the KK/compositeness scale interactions between the SM fields and the pseudoGoldstone become soft, so that the form factors are exponentially damped. The two scales entering the form factors are related to geometrical quantities in 5D:

$$
M_{\mathrm{KK}}=\frac{1}{\int_{0}^{L} d y a^{-1}(y)} \quad f_{h}=\frac{1}{g_{5}\left(\int_{0}^{L} d y a^{-2}(y)\right)^{1 / 2}} .
$$

This paper is organized as follows. In Sec. II we introduce the formalism that allows us to determine the spectrum of the 5D theory in a background-independent manner. We use these results in Sec. III to compute the one-loop effective potential for the holographic pseudoGoldstone boson. In Sec. IV we discuss how this formalism can applied for predicting the electroweak observables, such as the Higgs boson mass.

\section{5D FRAMEWORK}

We are interested in 4D strongly coupled theories, with the global symmetry group $G$ broken to a subgroup $H$ by strong interactions. The subgroup $K$ of the surviving global symmetry is weakly gauged by external vector fields (in phenomenological applications, $K$ is typically the SM gauge group). The 4D setup contains also chiral fermions coupled to composite operators of the strong sector. We describe below a 5D model with analogous properties.

We consider 5D gauge theories with the gauge group $G$. Four dimensions are flat and noncompact, while the fifth dimension is an interval, $x_{5} \in[0, L]$. The gravitational background is curved because of the warp factor $a\left(x_{5}\right)$. The line element is

$$
d s^{2}=a^{2}\left(x_{5}\right) \eta_{\mu \nu} d x^{\mu} d x^{\nu}-d x_{5}^{2} .
$$

We fix $a(0)=1$. The choice $a\left(x_{5}\right)=1$ corresponds to 5D flat space, while $a\left(x_{5}\right)=e^{-k x_{5}}$ corresponds to a slice of $\mathrm{AdS}_{5}$. For most of the subsequent discussion we do not specify the warp factor. We only assume that it is a monotonic and nonincreasing function, so that it makes sense to talk about a UV brane at $x_{5}=0$ and an IR brane at $x_{5}=L$, where the value of the warp factor is $a(L) \equiv a_{L} \leq 1$.

The bulk contains gauge fields $A_{M}=A_{M}^{\alpha} T^{\alpha}$, where $T^{\alpha}$ are Hermitian generators of the fundamental representation of $G$. We also introduce bulk fermions $\psi$, which transform under a representation $t^{\alpha}$ of $G$. The 5D action reads

$$
\begin{aligned}
S_{5 \mathrm{D}}= & \int d^{4} x \int_{0}^{L} d x_{5} \sqrt{g}\left(-\frac{1}{2 g_{5}^{2}} \operatorname{Tr}\left\{F_{M N} F^{M N}\right\}\right. \\
& \left.+\bar{\psi}\left(i \Gamma^{N} D_{N}-M\right) \psi\right)
\end{aligned}
$$

where $D_{N}=\partial_{N}-i A_{N}^{\alpha} t^{\alpha}$ and $g_{5}$ is the 5D dimensionful gauge coupling.

The 5D gauge symmetry is broken down to the subgroup $H \subset G$ on the IR brane and to $K \subset H$ on the UV brane. To simplify the subsequent discussion and reduce the number of indices we henceforth $\operatorname{set}^{1} K=H$. We divide the generators of $G$ as follows. The generators of $H$ are denoted by $T^{a}$ and $t^{a}$, while the generators from the coset $G / H$ are denoted by $T^{\hat{a}}$ and $t^{\hat{a}}$. The commutation relations can be written as ${ }^{2}$

$$
\begin{gathered}
{\left[T^{a}, T^{b}\right]=i f^{a b c} T^{c}, \quad\left[T^{a}, T^{\hat{b}}\right]=i f^{a \hat{b}} \hat{c} T^{\hat{c}},} \\
{\left[T^{\hat{a}}, T^{\hat{b}}\right]=i f^{\hat{a} \hat{b}} T^{c} .}
\end{gathered}
$$

The fermionic representation is divided as $\psi=\left(\psi^{m}, \psi^{\hat{m}}\right)$, in such a way that the generators satisfy $\left(t^{a}\right)_{m \hat{n}}=\left(t^{a}\right)_{\hat{m} n}=$ 0 and $\left(t^{\hat{a}}\right)_{m n}=\left(t^{a}\right)_{\hat{m} \hat{n}}=0$. We impose the boundary conditions

$$
\partial_{5} A_{\mu}^{a}=A_{\mu}^{\hat{a}}=A_{5}^{a}=\psi_{R}^{m}=\psi_{L}^{\hat{m}}=0, \quad x_{5}=0, L .
$$

One result of such boundary conditions is that the low energy theory contains 4D scalar excitations $h^{\hat{a}}$, which originate from $A_{5}^{\hat{a}}$. These scalars have a flat potential at

\footnotetext{
${ }^{1}$ Models with $K=H$ cannot be fully realistic. First, in such case the Weinberg angle cannot be made consistent with experiment [13]. Second, consistency with electroweak precision measurements typically requires $H$ to include custodial symmetry. However our results can be easily extended to the realistic case $K=G_{\text {SM }} \subset H$.

${ }^{2}$ To simplify subsequent computations we assumed that we can assign $Z_{2}$ parity to the generators, such that the $H$ generators are even and the coset generators are odd. This implies that the structure constants $f^{a b c}$ and $f^{\hat{a} \hat{b} \hat{c}}$ vanish. Our results remain unchanged when $f^{\hat{a} \hat{b} \hat{c}} \neq 0$.
} 
tree level and they can be interpreted as a dual description of the Goldstone bosons arising after spontaneous breaking of the global symmetry in 4D. There are also zero modes corresponding to $A_{\mu}^{a}, \psi_{L}^{m}$ and $\psi_{R}^{\hat{m}}$. They are interpreted as external fundamental fields mixing with composite states of the 4D strong sector. These zero modes are all massless as long as the scalar $h^{\hat{a}}$ does not develop a vev.

Our goal is to determine the spectrum of the theory in the presence of the vev $h=\left\langle\left(h^{\hat{a}} h^{\hat{a}}\right)^{1 / 2}\right\rangle$. To this end we rewrite the $5 \mathrm{D}$ theory in the KK basis

$$
\begin{aligned}
A_{\mu}^{a}\left(x, x_{5}\right) & =\sum_{n} f_{n}^{a}\left(x_{5}, h\right) A_{\mu, n}(x) \\
A_{5}^{a}\left(x, x_{5}\right) & =\sum_{n} \frac{\partial_{5} f_{n}^{a}\left(x_{5}, h\right)}{m_{n}(h)} h_{n}(x) \\
A_{\mu}^{\hat{a}}\left(x, x_{5}\right) & =\sum_{n} f_{n}^{\hat{a}}\left(x_{5}, h\right) A_{\mu, n}(x) \\
A_{5}^{\hat{a}}\left(x, x_{5}\right) & =\frac{C_{h}}{a^{2}\left(x_{5}\right)} h^{\hat{a}}(x)+\sum_{n} \frac{\partial_{5} f_{n}^{\hat{a}}\left(x_{5}, h\right)}{m_{n}(h)} h_{n}(x) \\
\psi_{L}^{m}\left(x, x_{5}\right) & =\sum_{n} f_{L, n}^{m}\left(x_{5}, h\right) \psi_{L, n}(x) \\
\psi_{R}^{m}\left(x, x_{5}\right) & =\sum_{n} f_{R, n}^{m}\left(x_{5}, h\right) \psi_{R, n}(x) \\
\psi_{L}^{\hat{m}}\left(x, x_{5}\right) & =\sum_{n} f_{L, n}^{\hat{m}}\left(x_{5}, h\right) \psi_{L, n}(x) \\
\psi_{R}^{\hat{m}}\left(x, x_{5}\right) & =\sum_{n} f_{R, n}^{\hat{m}}\left(x_{5}, h\right) \psi_{R, n}(x) .
\end{aligned}
$$

Above we have singled out the scalars $h^{\hat{a}}(x)$ and the corresponding KK profile. The normalization constant should be chosen as $C_{h}=g_{5}\left(\int_{0}^{L} a^{-2}\right)^{-1 / 2}$ so that the scalars are canonically normalized. The KK profiles $f_{n}\left(x_{5}, h\right)$ should be chosen such that the $5 \mathrm{D}$ action is rewritten in terms of a tower of $4 \mathrm{D}$ fields $A_{n}(x)$ and $\psi_{n}(x)$ whose kinetic and mass terms are diagonal in $n$ :

$$
\begin{aligned}
S_{5 \mathrm{D}}= & \int d^{4} x\left\{\frac{1}{2}\left(\partial_{\mu} h^{\hat{a}}\right)^{2}+\sum_{n}\left(-\frac{1}{4}\left[\partial_{\mu} A_{\nu, n}-\partial_{\nu} A_{\mu, n}\right]^{2}\right.\right. \\
& \left.\left.+\frac{1}{2} m_{n}^{2}(h) A_{\mu, n}^{2}\right)+\sum_{n} \bar{\psi}_{n}\left[\gamma^{\mu} \partial_{\mu}-\tilde{m}_{n}(h)\right] \psi_{n}+\ldots\right\}
\end{aligned}
$$

(the dots stand for cubic and quartic interactions and for gauge bosons kinetic mixing with the Goldstones.) This is achieved if the profiles solve the equations of motion in the presence of the scalar vev, and satisfy the boundary conditions

$$
\begin{aligned}
\partial_{5} f_{n}^{a}\left(x_{5}, h\right) & =f_{n}^{\hat{a}}\left(x_{5}, h\right)=f_{R, n}^{m}\left(x_{5}, h\right)=f_{L, n}^{\hat{m}}\left(x_{5}, h\right)=0, \\
x_{5} & =0, L .
\end{aligned}
$$

The profiles also satisfy normalization conditions, which are not important in what follows.
The equations of motion in the presence of the scalar vev are complicated, as they mix Neumann and Dirichlet modes. However, the 5D gauge invariance relates them to the solutions with $h=0$. For the gauge fields we have

$$
f^{\alpha}\left(x_{5}, h\right) T^{\alpha}=\omega^{-1}\left(x_{5}, h\right) f^{\alpha}\left(x_{5}, 0\right) T^{\alpha} \omega\left(x_{5}, h\right),
$$

where $\omega\left(x_{5}, h\right)$ is the gauge transformations that removes the vev of $h^{\hat{a}}$ :

$$
\omega\left(x_{5}, h\right)=\exp \left(-i C_{h} h^{\hat{a}} T^{a} \int_{0}^{x_{5}} d y a^{-2}(y)\right) .
$$

For $h=0$ the gauge KK profiles satisfy a simple equation of motion:

$$
\left(\partial_{5}^{2}+2 \frac{a^{\prime}}{a} \partial_{5}+\frac{m_{n}^{2}}{a^{2}}\right) f_{n}^{\alpha}\left(x_{5}, 0\right)=0
$$

which is the same for the Dirichlet and the Neumann modes. We denote the two independent solutions by $C\left(x_{5}, m_{n}\right)$ and $S\left(x_{5}, m_{n}\right)$. We choose them such that they satisfy the initial conditions $C(0, z)=1, C^{\prime}(0, z)=0$, $S(0, z)=0, S^{\prime}(0, z)=z$. These base functions can be viewed as a warped generalization of the cosine and the sine function (in flat 5D $C=\cos \left(x_{5} z\right), S=\sin \left(x_{5} z\right)$ ). Then the gauge KK profiles can we written as

$$
f_{n}^{a}\left(x_{5}, 0\right)=C_{n, a} C\left(x_{5}, m_{n}\right) \quad f_{n}^{\hat{a}}\left(x_{5}, 0\right)=C_{n, \hat{a}} S\left(x_{5}, m_{n}\right) .
$$

We can now derive $f_{n}^{\alpha}\left(x_{5}, h\right)$ using Eq. (11). Note that the resulting profiles satisfy the UV boundary conditions (10). This is because we have chosen the transformation matrix $\omega$ in such a way that, for $x_{5}=0, f_{n}^{\alpha}\left(x_{5}, 0\right)=f_{n}^{\alpha}\left(x_{5}, h\right)$. Now, the IR boundary conditions fix the quantization of the KK masses $m_{n}(h)$. After some group algebra we obtain

$$
\begin{aligned}
& \cos ^{2}\left(\lambda_{r} h / f_{h}\right) C^{\prime}\left(L, m_{n}\right) S\left(L, m_{n}\right) \\
& +\sin ^{2}\left(\lambda_{r} h / f_{h}\right) S^{\prime}\left(L, m_{n}\right) C\left(L, m_{n}\right)=0,
\end{aligned}
$$

where the "Higgs decay constant" is defined as

$$
f_{h}^{2}=\frac{1}{g_{5}^{2} \int_{0}^{L} d y a^{-2}(y)}
$$

and $\left(\lambda_{r} h\right)^{2}$ are eigenvalues of the symmetric matrix $\mathcal{M}$ :

$$
\mathcal{M}_{a b}=h^{\hat{a}} h^{\hat{b}} f^{a \hat{a} \hat{c}} f^{b \hat{b} \hat{c}} .
$$

Furthermore, using the Wronskian relation

$$
S^{\prime}\left(x_{5}, z\right) C\left(x_{5}, z\right)-C^{\prime}\left(x_{5}, z\right) S\left(x_{5}, z\right)=z a^{-2}\left(x_{5}\right)
$$

we can rewrite the quantization condition in a more convenient form:

$$
\begin{aligned}
1+F_{(1)}\left(m_{n}^{2}\right) \sin ^{2}\left(\frac{\lambda_{r} h}{f_{h}}\right) & =0, \\
F_{(1)}\left(z^{2}\right) & =\frac{z}{a_{L}^{2} C^{\prime}(L, z) S(L, z)} .
\end{aligned}
$$


The set of discrete numbers $\lambda_{r}$ depends on the gauge groups $G$ and $H$ and $r$ runs over all zero mode gauge bosons. For example, for $G=S U(3), H=S U(2) \times U(1)$ from Eq. (17) we find $\lambda_{1}=0$ (the photon), $\lambda_{2}=\lambda_{3}=1 / 2$ (the $W$ bosons), $\lambda_{4}=1$ (the $Z$ boson with a wrong Weinberg angle). ${ }^{3}$

Proceeding analogously with fermionic KK profiles we obtain

$$
\begin{aligned}
1+F_{(1 / 2)}\left(m_{n}^{2}\right) \sin ^{2}\left(\frac{\lambda_{r} h}{f_{h}}\right) & =0, \\
F_{(1 / 2)}\left(z^{2}\right) & =-\frac{1}{S_{M}(L, z) S_{-M}(L, z)} .
\end{aligned}
$$

Here $S_{M}\left(x_{5}, z\right)$ is a solution of the differential equation

$$
\left[\partial_{5}^{2}+\left(\frac{a^{\prime}}{a}+2 M\right) \partial_{5}+\frac{z^{2}}{a^{2}}\right] f=0
$$

that satisfies the boundary conditions $S_{M}(0, z)=0$, $S_{M}^{\prime}(0, z)=z$, and $\left(\lambda_{r} h\right)^{2}$ denote eigenvalues of the matrix:

$$
\mathcal{M}_{m n}=h^{\hat{a}} h^{\hat{b}}\left(t^{\hat{a}}\right)_{m \hat{n}}\left(t^{\hat{b}}\right)_{\hat{n} n} .
$$

The Eqs. (19) and (20) are the starting point for computing the one-loop effective potential for the scalars $h^{\hat{a}}$.

\section{ONE-LOOP POTENTIAL FOR THE PSEUDO-GOLDSTONE}

\section{A. Coleman-Weinberg potential in 5D}

The Coleman-Weinberg formula in 5D KK theories takes the form

$$
V=\frac{N}{2} \sum_{n} \int \frac{d^{4} p}{(2 \pi)^{4}} \log \left(p^{2}+m_{n}^{2}\right) .
$$

Here $N$ is a number of degrees of freedom of a given particle and the sum goes over an infinite KK tower. We can regulate the $4 \mathrm{D}$ momentum integral using the dimensional regularization, with $d=4+\epsilon$ :

$$
V=\frac{N}{(4 \pi)^{d / 2} \Gamma(d / 2)} \sum_{n} \int d p p^{d-1} \log \left(1+\frac{m_{n}^{2}}{p^{2}}\right) .
$$

In the 5D framework the pseudo-Goldstone boson potential is finite, so the result does not depend on the way we regularize integrals. Now, in dimensional regularization we can evaluate the $4 \mathrm{D}$ momentum integral:

\footnotetext{
${ }^{3}$ The correct $Z$ boson mass can be adjusted by adding a $U(1)_{X}$ factor to $G$ and $H$ and breaking one combinations of $U(1)$ 's by UV boundary conditions. The quantization conditions are very similar in this case, only the form factor for the $Z$ boson is multiplied by a number that depends on the mixing angle between the $U(1)$ 's.
}

$$
V=\frac{N}{(4 \pi)^{d / 2}} \frac{\pi}{d \Gamma(d / 2) \sin (\pi d / 2)} \sum_{n} m_{n}^{d} .
$$

Using techniques of holomorphic functions, the sum over the KK modes can be traded for an integral. To this end we need a spectral function $\rho\left(z^{2}\right)$, which is holomorphic in the $\operatorname{Rez}>0$ part of the complex plane and whose zeros on the real axis encode the KK spectrum, $\rho\left(m_{n}^{2}\right)=0$. Having this, we can rewrite the sum as (see e.g. [7])

$$
\sum_{n} m_{n}^{d}=\frac{d \sin (\pi d / 2)}{\pi} \int_{0}^{\infty} d y y^{d-1} \log \rho\left(-y^{2}\right) .
$$

The Coleman-Weinberg potential thus becomes

$$
V=\frac{N}{(4 \pi)^{d / 2} \Gamma(d / 2)} \int_{0}^{\infty} d k k^{d-1} \log \rho\left(-k^{2}\right) .
$$

We showed in the previous section that, in the 5D framework, the spectral function encoding the KK spectrum in the presence of the pseudo-Goldstone vev is given by

$$
\rho\left(z^{2}\right)=1+F\left(z^{2}\right) \sin ^{2}\left(\frac{\lambda_{r} h}{f_{h}}\right),
$$

with some form factor $F\left(z^{2}\right)$ that depends on the 5D gravitational background (for fermions, also on bulk masses). At this point we can safely remove the regulator and set $d=4$. So finally, the scalar potential for the pseudo-Goldstone can be written as

$$
V(h)=\sum_{r} \frac{N_{r}}{(4 \pi)^{2}} \int_{0}^{\infty} d p p^{3} \log \left(1+F_{r}\left(-p^{2}\right) \sin ^{2}\left(\lambda_{r} h / f_{h}\right)\right) .
$$

The sum now goes over all gauge and fermionic zero modes; $N_{r}=+3$ for gauge bosons, $N_{r}=-4$ for fermions. The form factor $F_{(1)}$ for gauge bosons is given by Eq. (19), $F_{(1 / 2)}$ for fermions is given by Eq. (20).

The scalar potential (29) has the form we would expect in 4D theories with a composite pseudo-Goldstone boson arising as a consequence of spontaneous global symmetry breaking in a strongly interacting sector. In that setup the form factor describes an effective interaction vertex between the pseudo-Goldstone and fundamental particles in a low energy theory below the compositeness scale. The 5D theory predicts the same form on the pseudo-Goldstone potential, which makes the holographic interpretation possible. The advantage of the 5D framework is that also the form factor $F\left(z^{2}\right)$ is calculable, once we can solve the 5D equations of motion. In the remainder of this section we study the shape of the form factors predicted by $5 \mathrm{D}$ theories.

\section{B. Properties of the form factors}

Even without assuming a specific warp factor $a\left(x_{5}\right)$ and finding explicit solutions to the equations of motion we are able to say a lot about the behavior of the form factors in 
various energy regimes. It turns out that for an arbitrary warp factor the equations of motion can be formally solved below and above the KK scale $M_{\mathrm{KK}}$, where $M_{\mathrm{KK}}$ roughly equals the mass of the lightest resonance.

(1) Low-energy behavior. We first investigate the form factors at small momenta. Thus, we consider the equations of motion (13) and (21) with the $z^{2} / a^{2}$ term small enough to be treated perturbatively. In this regime the base solutions can be written as

$$
\begin{aligned}
C\left(x_{5}, z\right) & =1-z^{2} \int_{0}^{x_{5}} d y y a^{-2}(y)+\mathcal{O}\left(z^{4}\right) \\
S\left(x_{5}, z\right) & =z \int_{0}^{x_{5}} d y a^{-2}(y)+\mathcal{O}\left(z^{3}\right) \\
S_{M}\left(x_{5}, z\right) & =z \int_{0}^{x_{5}} d y a^{-1}(y) e^{-2 M y}+\mathcal{O}\left(z^{3}\right) .
\end{aligned}
$$

The form factors can be approximated by

$$
\begin{gathered}
F_{(1)}\left(-p^{2}\right) \approx \frac{g^{2} f_{h}^{2}}{p^{2}} \quad g=\frac{g_{5}}{\sqrt{L}} \\
F_{(1 / 2)}\left(-p^{2}\right) \approx \frac{y^{2} f_{h}^{2}}{p^{2}} \\
y^{2}=\frac{L \int_{0}^{L} d y a^{-2}(y)}{\int_{0}^{L} d y a^{-1}(y) e^{-2 M y} \int_{0}^{L} d y, a^{-1}(y) e^{2 M y}} .
\end{gathered}
$$

As could be expected, below the KK scale the form factors exhibit the $1 / p^{2}$ momentum dependence characteristic of pointlike particles. From Eqs. (19) and (20) the couplings $g$ and $y$ are related to the mass of the light particles:

$$
\begin{array}{ll}
m_{r}^{2} \approx g^{2} f_{h}^{2} \sin ^{2}\left(\frac{\lambda_{r} h}{f}\right)+\mathcal{O}\left(m_{r}^{4} / M_{\mathrm{KK}}^{2}\right) & \text { gauge } \\
m_{r}^{2} \approx y^{2} f_{h}^{2} \sin ^{2}\left(\frac{\lambda_{r} h}{f}\right)+\mathcal{O}\left(m_{r}^{4} / M_{\mathrm{KK}}^{2}\right) & \text { fermion. }
\end{array}
$$

(2) Intermediate-energy behavior. Next, we consider momenta above the KK scale (we will define it shortly). In the equations of motion we assume that the $z^{2} / a^{2}$ term is sizable close to $x_{5} \sim L$, while it remains a small perturbation for $x_{5} \sim 0$. Close to the UV brane the base solutions are still well approximated by Eq. (30). Close to the IR brane we assume that the $\partial_{5}$ term in the equations of motion can be neglected. Under this assumption the base solutions for $x_{5} \sim L$ can be written as

$$
\begin{gathered}
C\left(x_{5}, z\right) \approx a^{-1 / 2}\left(x_{5}\right) \alpha \cos \left(z \int_{0}^{x_{5}} a^{-1}(y)+\phi_{c}\right) \\
S\left(x_{5}, z\right) \approx a^{-1 / 2}\left(x_{5}\right) \beta \sin \left(z \int_{0}^{x_{5}} a^{-1}(y)+\phi_{s}\right) \\
S_{M}\left(x_{5}, z\right)=\beta_{M} e^{-M x_{5}} \sin \left(z \int_{0}^{x_{5}} a^{-1}(y)+\phi_{M}\right)
\end{gathered}
$$

This approximation is valid for $x_{5}$ satisfying $\left|a^{\prime}\left(x_{5}\right)\right| \ll|z|,|M| \ll z$. The amplitude and the phase shifts depend on the detailed behavior of the solutions in the crossover region and cannot be determined by our general analysis. They satisfy though the useful relations: $\beta \alpha \cos \left(\phi_{c}-\phi_{s}\right)=1$ and $\beta_{M} \beta_{-M} \cos \left(\phi_{M}-\phi_{-M}\right)=1$. The former follows from the Wronskian (18), while the latter from the Crowian:

$$
\begin{aligned}
& a^{2}\left(x_{5}\right) S_{M}^{\prime}\left(x_{5}, z\right) S_{-M}^{\prime}\left(x_{5}, z\right) \\
& \quad+z^{2} S_{M}\left(x_{5}, z\right) S_{-M}\left(x_{5}, z\right)=z^{2} .
\end{aligned}
$$

The form factors in the intermediate regime can be approximated by

$$
\begin{aligned}
& F_{(1)}\left(z^{2}\right) \approx-\frac{\cos \left(\phi_{c}-\phi_{s}\right)}{\sin \left(\int_{0}^{L} a^{-1}(y) z+\phi_{c}\right) \sin \left(\int_{0}^{L} a^{-1}(y) z+\phi_{s}\right)} \\
& F_{(1 / 2)}\left(z^{2}\right) \approx-\frac{\cos \left(\phi_{M}-\phi_{-M}\right)}{\sin \left(z \int_{0}^{L} a^{-1}(y)+\phi_{M}\right) \sin \left(z \int_{0}^{L} a^{-1}(y)+\phi_{-M}\right)} .
\end{aligned}
$$

It follows that

$$
\begin{aligned}
F_{(1)}\left(-k^{2}\right) & \approx F_{(1 / 2)}\left(-k^{2}\right) \approx e^{-2 k / M_{\mathrm{KK}}}, \\
M_{\mathrm{KK}} & =\frac{1}{\int_{0}^{L} d y a^{-1}(y)} .
\end{aligned}
$$

Above the KK scale the form factors are exponentially damped. From Eqs. (19) and (20) the KK is related to the mass of the lightest KK excitations:

$$
m_{1} \approx M_{\mathrm{KK}}(\pi-\phi) .
$$

(3) High-energy behavior. For very high momenta the warping of the 5D spacetime should no longer be visible. In the equations of motion this flatlike regime occurs for large enough $z$, such that the $\partial_{5}$ term can be neglected in the whole interval, including $x_{5} \sim 0$. Then the approximate base solutions are given by: 


$$
\begin{aligned}
C\left(x_{5}, z\right) & \approx a^{-1 / 2}\left(x_{5}\right) \cos \left(z \int_{0}^{x_{5}} a^{-1}(y)\right) \\
S\left(x_{5}, z\right) & \approx a^{-1 / 2}\left(x_{5}\right) \sin \left(z \int_{0}^{x_{5}} a^{-1}(y)\right) \\
S_{M}\left(x_{5}, z\right) & \approx e^{-M x_{5}} \sin \left(z \int_{0}^{x_{5}} a^{-1}(y)\right) .
\end{aligned}
$$

They are similar to the ones in the intermediate regime, but now the amplitudes and the phase shifts can be fixed by the boundary conditions at $x_{5}=0$. The form factors are given by

$$
\begin{gathered}
F_{(1)}\left(z^{2}\right) \approx-\frac{1}{\sin ^{2}\left(\int_{0}^{L} a^{-1}(y) z\right)} \\
F_{(1 / 2)}\left(z^{2}\right) \approx-\frac{1}{\sin ^{2}\left(z \int_{0}^{L} a^{-1}(y)\right)} .
\end{gathered}
$$

For $z^{2}=-k^{2}$ the form factors exhibit an exponential damping, as in Eq. (36). This ensures the convergence of the momentum integral and, in consequence, a finiteness of the pseudo-Goldstone potential at one loop.

We can easily write a function that interpolates between the $1 / k^{2}$ behavior at low energy and the exponential damping above the KK scale:

$$
\begin{aligned}
F_{(1)}\left(-p^{2}\right) & \approx \frac{g^{2} f_{h}^{2}}{M_{\mathrm{KK}}^{2} \sinh ^{2}\left(p / M_{\mathrm{KK}}\right)} \\
F_{(1 / 2)}\left(-p^{2}\right) & \approx \frac{y^{2} f_{h}^{2}}{M_{\mathrm{KK}}^{2} \sinh ^{2}\left(p / M_{\mathrm{KK}}\right)} .
\end{aligned}
$$

This naive guess works astonishingly well in many cases. We will see the accuracy of Eq. (40) by comparing the approximate with the exact form factors derived by solving the equations of motion in specific backgrounds. The approximation of the fermionic warp factor is less accurate for large bulk masses. This could be improved, but large bulk masses are not important in practice, as they lead to suppressed contributions to the pseudo-Goldstone potential.

\section{Exact form factors}

Below, we give a few examples of solutions to the equations of motion in specific backgrounds and we calculate the corresponding form factors.

\section{Flat spacetime}

For the 5D Minkowski spacetime we set $a\left(x_{5}\right)=1$. As $a(L) / a(0)=1$ this background does not have a holo- graphic interpretation, but it is the simplest one to perform 5D computations. by

The base functions solving Eqs. (13) and (21) are given

$$
\begin{gathered}
C\left(x_{5}, z\right)=\cos \left(z x_{5}\right) \quad S\left(x_{5}, z\right)=\sin \left(z x_{5}\right) \\
S_{M}\left(x_{5}, z\right)=\frac{z e^{-M x_{5}}}{\sqrt{z^{2}-M^{2}}} \sin \left(\sqrt{z^{2}-M^{2}} x_{5}\right) .
\end{gathered}
$$

Inserting this into Eqs. (19) and (20) we obtain [5]

$$
\begin{aligned}
F_{(1)}\left(-p^{2}\right) & =\frac{1}{\sinh ^{2}(p L)} \\
F_{(1 / 2)}\left(-p^{2}\right) & =\frac{p^{2}+M^{2}}{p^{2}} \frac{1}{\sin ^{2}\left(\sqrt{p^{2}+M^{2}} L\right)} .
\end{aligned}
$$

In this case our approximate expression (40) for the gauge form factor, with $g f_{h}=M_{\mathrm{KK}}=1 / L$, is equal to the true. The fermionic approximation is fine for $M L \ll 1$.

\section{AdS $_{5}$ spacetime}

We take $a\left(x_{5}\right)=e^{-k x_{5}}$ and, customarily, we parametrize the bulk fermion mass as $M=c k$. This is the RandallSundrum spacetime [14], which arises when the background is shaped by 5D gravity with a negative cosmological constant, $V=-6 k^{2}$. 5D theories in such background are conjectured to be dual to 4D theories, with a conformally symmetric strong sector [3]. The parameter $c$ is related to an anomalous dimension of the fermionic composite operators, to which the fundamental fermions are coupled [15].

Solutions to Eqs. (13) and (21) can be expressed in terms of Bessel functions [16]. The base functions with prescribed UV boundary conditions are given by the following expressions:

$$
\begin{aligned}
& C\left(x_{5}, z\right)=\frac{\pi z}{2 k} a^{-1}\left(x_{5}\right)\left[Y_{0}\left(\frac{z}{k}\right) J_{1}\left(\frac{z}{k a\left(x_{5}\right)}\right)\right. \\
& \left.-J_{0}\left(\frac{z}{k}\right) Y_{1}\left(\frac{z}{k a\left(x_{5}\right)}\right)\right] \\
& S\left(x_{5}, z\right)=\frac{\pi z}{2 k} a^{-1}\left(x_{5}\right)\left[J_{1}\left(\frac{z}{k}\right) Y_{1}\left(\frac{z}{k a\left(x_{5}\right)}\right)\right. \\
& \left.-Y_{1}\left(\frac{z}{k}\right) J_{1}\left(\frac{z}{k a\left(x_{5}\right)}\right)\right] \\
& S_{M}\left(x_{5}, z\right)=\frac{\pi z}{2 k} a^{c-(1 / 2)}\left(x_{5}\right)\left[J_{(1 / 2)-c}\left(\frac{z}{k}\right) Y_{(1 / 2)-c}\left(\frac{z}{k a\left(x_{5}\right)}\right)\right. \\
& \left.-Y_{(1 / 2)-c}\left(\frac{z}{k}\right) J_{(1 / 2)-c}\left(\frac{z}{k a\left(x_{5}\right)}\right)\right] \text {. }
\end{aligned}
$$

The form factors are given by $[7,10]$

$$
F_{(1)}\left(-p^{2}\right)=\frac{a_{L} k^{2}}{p^{2}} \frac{1}{\left[I_{0}\left(\frac{p}{k}\right) K_{0}\left(\frac{p}{k a_{L}}\right)-K_{0}\left(\frac{p}{k}\right) I_{0}\left(\frac{p}{k a_{L}}\right)\right]\left[I_{1}\left(\frac{p}{k}\right) K_{1}\left(\frac{p}{k a_{L}}\right)-K_{1}\left(\frac{p}{k}\right) I_{1}\left(\frac{p}{k a_{L}}\right)\right]}
$$


$F_{(1 / 2)}\left(-p^{2}\right)=\frac{a_{L} k^{2}}{p^{2}} \frac{1}{\left[I_{(1 / 2)-c}\left(\frac{p}{k}\right) K_{(1 / 2)-c}\left(\frac{p}{k a_{L}}\right)-K_{(1 / 2)-c}\left(\frac{p}{k}\right) I_{(1 / 2)-c}\left(\frac{p}{k a_{L}}\right)\right]\left[I_{(1 / 2)+c}\left(\frac{p}{k}\right) K_{(1 / 2)+c}\left(\frac{p}{k a_{L}}\right)-K_{(1 / 2)+c}\left(\frac{p}{k}\right) I_{(1 / 2)+c}\left(\frac{p}{k a_{L}}\right)\right]}$.

These form factors can be approximated by those in Eq. (40) with

$$
\begin{gathered}
\left(g f_{h}\right)^{2}=\frac{2 k^{2}}{k L\left(a_{L}^{-2}-1\right)} \quad M_{\mathrm{KK}}=\frac{k}{\left(a_{L}^{-1}-1\right)} \\
\left(y f_{h}\right)^{2}=\frac{(2 c+1)(-2 c+1) k^{2}}{\left(a_{L}^{2 c-1}-1\right)\left(a_{L}^{-2 c-1}-1\right)} .
\end{gathered}
$$

The accurateness of these approximations is visualized in Fig. 1. The approximate of the gauge form factor precisely traces the true one. For fermions the approximate is even better for $|c| \leq 1 / 2$, while it deviates for $|c| \gg 1 / 2$.

\section{Dilatonic spacetime}

One more example where the gauge equation of motion can be solved (the fermionic one is more resistant) is the $5 \mathrm{D}$ spacetime with the warp factor varying as a power of $x_{5}$ :

$$
a\left(x_{5}\right)=\left(1-\frac{k x_{5}}{\gamma-1}\right)^{\gamma}
$$

For definiteness we assume $\gamma>1$, so that $a_{L}<1$ for positive $k$. Of course, we need $k L<\gamma-1$ to avoid a naked singularity. In the conformal limit $\gamma \rightarrow \infty$ we recover the exponential warp factor of $\mathrm{AdS}_{5}$. For finite $\gamma$ the $5 \mathrm{D}$ model is dual to $4 \mathrm{D}$ strongly coupled theories with conformal symmetry explicitly broken by a coupling $\sim 1 / \gamma$ that logarithmically increases towards IR.

This power-law warp factor arises as a solution in 5D gravity-dilaton theories with the dilaton potential

$$
V(\phi)=-6 k^{2} \frac{\gamma(\gamma-1 / 4)}{(\gamma-1)^{2}} e^{ \pm(2 / \sqrt{3} \gamma)\left(\phi-\phi_{0}\right)}
$$

and appropriate boundary potentials. The well-known example where such background appears is the HoravaWitten model [17] compactified to 5D [18], in which case $\gamma=1 / 6$.

Solutions to Eq. (13) can be written, much as in the $\mathrm{AdS}_{5}$ case, in terms of the Bessel functions,

$$
\begin{aligned}
& C\left(x_{5}, z\right)=\frac{\pi z}{2 k} a^{-1+(1 / 2 \gamma)}\left[Y_{1 /(2 \gamma-2)}\left(\frac{z}{k}\right) J_{(2 \gamma-1) /(2 \gamma-2)}\left(\frac{z}{k a^{1-(1 / \gamma)}}\right)-J_{1 /(2 \gamma-2)}\left(\frac{z}{k}\right) Y_{(2 \gamma-1) /(2 \gamma-2)}\left(\frac{z}{k a^{1-(1 / \gamma)}}\right)\right] \\
& S\left(x_{5}, z\right)=\frac{\pi z}{2 k} a^{-1+(1 / 2 \gamma)}\left[J_{(2 \gamma-1) /(2 \gamma-2)}\left(\frac{z}{k}\right) Y_{(2 \gamma-1) /(2 \gamma-2)}\left(\frac{z}{k a^{1-(1 / \gamma)}}\right)-Y_{(2 \gamma-1) /(2 \gamma-2)}\left(\frac{z}{k}\right) J_{(2 \gamma-1) /(2 \gamma-2)}\left(\frac{z}{k a^{1-(1 / \gamma)}}\right)\right]
\end{aligned}
$$

The gauge form factor is readily calculated:

$$
\begin{aligned}
F_{(1)}\left(-p^{2}\right)= & \frac{a_{L}^{1-(1 / \gamma)} k^{2}}{p^{2}} \cdot \frac{1}{\left[I_{1 /(2 \gamma-2)}\left(\frac{p}{k}\right) K_{1 /(2 \gamma-2)}\left(\frac{p}{k a_{L}^{1-(1 / \gamma)}}\right)-K_{1 /(2 \gamma-2)}\left(\frac{p}{k}\right) I_{(2 \gamma-2)}\left(\frac{p}{\left.k a_{L}^{1-(1 / \gamma)}\right)}\right]\right.} \\
& \cdot \frac{1}{\left[I_{(2 \gamma-1) /(2 \gamma-2)}\left(\frac{p}{k}\right) I_{(2 \gamma-1) /(2 \gamma-2)}\left(\frac{p}{k a_{L}^{1-(1 / \gamma)}}\right)-K_{(2 \gamma-1) /(2 \gamma-2)}\left(\frac{p}{k}\right) I_{(2 \gamma-1) /(2 \gamma-2)}\left(\frac{p}{\left.\left.k a_{L}^{1-(1 / \gamma)}\right)\right]}\right.\right.}
\end{aligned}
$$
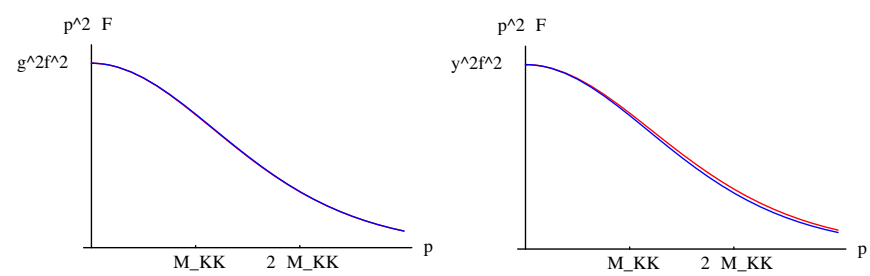

FIG. 1 (color online). Left panel: the true (red) and the approximate (blue) gauge form factor in $\mathrm{AdS}_{5}$ for $a_{L}=10^{-3}$. If you see only one line, it is because the approximate is so successful. Right panel: the same for the fermionic form factor, with $c=0.7$.
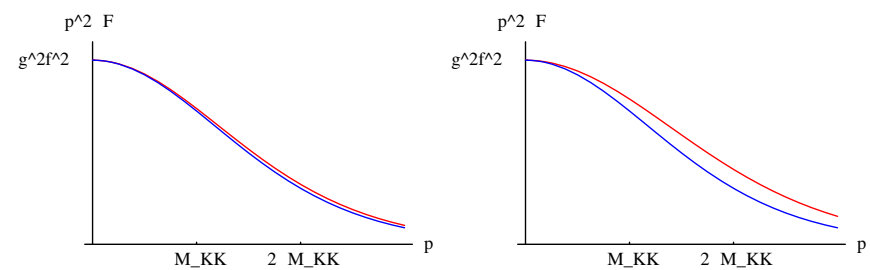

FIG. 2 (color online). Left panel: the true (red) and the approximate (blue) gauge form factor in the dilatonic spacetime, for $a_{L}=10^{-3}$ and $\gamma=4$. Right panel: the same for $\gamma=1.5$. 
The approximate expression is that of Eq. (40) with

$$
\begin{aligned}
\left(g f_{h}\right)^{2} & =\frac{(2 \gamma-1) k^{2}}{(\gamma-1) k L\left(a_{L}^{-2+1 / \gamma}-1\right)} \\
M_{\mathrm{KK}} & =\frac{k}{a_{L}^{-1+1 / \gamma}-1} .
\end{aligned}
$$

As can be seen from Fig. 2, the approximate expression works very well as long as $\gamma$ is sufficiently larger than 1 .

\section{DISCUSSION}

We presented a simple algorithm for computing the form factors entering the pseudo-Goldstone potential. The methods developed here clarify the existing computations in the flat and $\mathrm{AdS}_{5}$ spacetimes, and facilitate extensions to other 5D backgrounds. Our results should be relevant not only for electroweak breaking, but also for the so-called natural inflation [19], which also makes use of the Goldstone mechanism.

Furthermore, we have argued that the form factors obtained in the 5D framework can often be adequately approximated by Eq. (40). Consequently, the pseudoGoldstone potential can be approximated by:

$V(h) \approx \sum_{r} \frac{N_{r}}{(4 \pi)^{2}} \int_{0}^{\infty} d p p^{3} \log \left(1+\frac{g_{r}^{2} f_{h}^{2}}{M_{\mathrm{KK}}^{2}} \frac{\sin ^{2}\left(\lambda_{r} h / f_{h}\right)}{\sinh ^{2}\left(p / M_{\mathrm{KK}}\right)}\right)$,

where the sum goes over all zero modes. Recall that the two scales entering the potential are simply related to 5D geometrical quantities:

$$
M_{\mathrm{KK}}=\frac{1}{\int_{0}^{L} d y a^{-1}(y)}, \quad f_{h}^{2}=\frac{1}{g^{2} L \int_{0}^{L} d y a^{-2}(y)} .
$$

We conclude with a simple example showing how our results can be applied in phenomenological situations. In the following we identify the pseudo-Goldstone boson $h^{\hat{a}}$ with the Higgs field responsible for electroweak symmetry breaking of the SM. We study how various observables like the electroweak scale or the Higgs mass depend on the two scales defined in Eq. (53). We assume here that the Higgs potential (52) is dominated by a contribution of only one low energy particle species, the top quark. Then we can write the Higgs potential as

$$
V(h) \approx-\frac{3}{4 \pi^{2}} M_{\mathrm{KK}}^{4} \int_{0}^{\infty} d p p^{3} \log \left(1+\frac{y_{t}^{2} f_{h}^{2}}{M_{\mathrm{KK}}^{2}} \frac{\sin ^{2}\left(\lambda_{t} h / f_{h}\right)}{\sinh ^{2}(p)}\right) .
$$

At the origin, $V^{\prime \prime}(0)<0$, so the Higgs develops a vev and electroweak symmetry is broken. The minimum occurs at $\sin \left(\lambda_{t} h / f_{h}\right)=1$, thus $\langle h\rangle=\pi f_{h} / 2 \lambda_{t}$. Consequently, the top mass and the $W$ mass are given by

$$
m_{t}=y_{t} f_{h}, \quad m_{W}=g f_{h} \sin \left(\lambda_{W}\langle h\rangle / f_{h}\right) .
$$

The relation between the Higgs decay constant and the electroweak scale depends on group representations via $\lambda_{W}$, but typically $m_{W} \sim g f_{h}$, where $g$ is the $S U(2)_{W}$ gauge coupling constant. Thus, in this simple example it is not possible to separate the electroweak scale from the global symmetry breaking scale. Therefore, for the model to be phenomenologically relevant, we need to separate the KK scale from the electroweak scale $m_{W} \sim g f_{h} \ll M_{\mathrm{KK}}$. From Eq. (53) the ratio is given by

$$
\frac{M_{\mathrm{KK}}}{g f_{h}}=\frac{\left(L \int_{0}^{L} d y a^{-2}(y)\right)^{1 / 2}}{\int_{0}^{L} d y a^{-1}(y)} .
$$

For flat 5D spacetime this ratio is 1 , which is of course unacceptable. But, in general, warping leads to a desired scale separation. For the Randall-Sundrum model one finds that the scale separation is logarithmically enhanced $[7,8,10]$

$$
M_{\mathrm{KK}} / g f_{h} \approx \sqrt{k L / 2} \sim \log ^{1 / 2}\left(M_{\mathrm{Pl}} / M_{\mathrm{KK}}\right) .
$$

For the power-law warp factor (47) we find

$$
M_{\mathrm{KK}} / g f_{h} \approx \sqrt{k L(\gamma-1) /(2 \gamma-1)} a_{L}^{-1 / 2 \gamma}
$$

and even larger scale separation can be obtained for $a_{L} \ll$ 1 and $\gamma$ away from the conformal limit.

Computing the second derivative of Eq. (54) at the minimum we find the Higgs boson mass

$$
m_{h}^{2}=\frac{9}{4 \pi^{2}} \lambda_{t}^{2} m_{t}^{2} \frac{M_{\mathrm{KK}}^{2}}{f_{h}^{2}} \alpha\left(m_{t}^{2} / M_{\mathrm{KK}}^{2}\right),
$$

where $\alpha(x)=(2 / 3) \int_{0}^{\infty} t^{3}\left(\sinh ^{2} t+x\right)^{-1}$ is a slowly varying function $\sim 1 ; \alpha(0)=\zeta(3), \alpha(1)=(3 / 4) \zeta(3)$. The Higgs mass is suppressed with respect to the electroweak scale by a loop factor, but it is enhanced by the ratio $M_{\mathrm{KK}} / f_{h}$. Thus, enough separation between the KK and the electroweak scale automatically ensures that the Higgs boson mass is consistent with current experimental bounds. This conclusion does not depend whether the 5D background is conformal, as in the Randall-Sundrum model, or if it is of a more general warped form.

This example illustrates how the formalism we developed clarifies the dependence of the observables in the electroweak sector on the scales describing the 5D model or its holographic 4D dual. Of course, a full-fledged phenomenological analysis requires computing corrections to electroweak precision observables. This is left for future studies.

\section{ACKNOWLEDGMENTS}

I would like to thank Ricardo Rattazzi and Claudio Scrucca for nice discussions. I am partially supported by the European Community Contract No. MRTN-CT-2004503369 for the years 2004-2008 and by the MEiN Grant No. 1 P03B 09929 for the years 2005-2007. 
[1] D. B. Kaplan and H. Georgi, Phys. Lett. 136B, 183 (1984); M. J. Dugan, H. Georgi, and D. B. Kaplan, Nucl. Phys. B254, 299 (1985).

[2] J. M. Maldacena, Adv. Theor. Math. Phys. 2, 231 (1998); Int. J. Theor. Phys. 38, 1113 (1999).

[3] N. Arkani-Hamed, M. Porrati, and L. Randall, J. High Energy Phys. 08 (2001) 017; R. Rattazzi and A. Zaffaroni, J. High Energy Phys. 04 (2001) 021; M. Pérez-Victoria, J. High Energy Phys. 05 (2001) 064; T. Gherghetta, hep$\mathrm{ph} / 0601213$.

[4] Y. Hosotani, Phys. Lett. 126B, 309 (1983); Ann. Phys. (N.Y.) 190, 233 (1989); H. Hatanaka, T. Inami, and C. S. Lim, Mod. Phys. Lett. A 13, 2601 (1998).

[5] I. Antoniadis, K. Benakli, and M. Quiros, New J. Phys. 3, 20 (2001); M. Kubo, C. S. Lim, and H. Yamashita, Mod. Phys. Lett. A 17, 2249 (2002); L. J. Hall, Y. Nomura, and D. R. Smith, Nucl. Phys. B639, 307 (2002); G. Burdman and Y. Nomura, Nucl. Phys. B656, 3 (2003); C. Csaki, C. Grojean, and H. Murayama, Phys. Rev. D 67, 085012 (2003); C. A. Scrucca, M. Serone, and L. Silvestrini, Nucl. Phys. B669, 128 (2003); C. A. Scrucca, M. Serone, L. Silvestrini, and A. Wulzer, J. High Energy Phys. 02 (2004) 049; Y. Hosotani, S. Noda, and K. Takenaga, Phys. Lett. B 607, 276 (2005); G. Cacciapaglia, C. Csaki, and S.C. Park, J. High Energy Phys. 03 (2006) 099; G. Panico, M. Serone, and A. Wulzer, Nucl. Phys. B739, 186 (2006).

[6] R. Contino, Y. Nomura, and A. Pomarol, Nucl. Phys. B671, 148 (2003).
[7] K. y. Oda and A. Weiler, Phys. Lett. B 606, 408 (2005).

[8] K. Agashe, R. Contino, and A. Pomarol, Nucl. Phys. B719, 165 (2005).

[9] Y. Hosotani and M. Mabe, Phys. Lett. B 615, 257 (2005).

[10] Y. Hosotani, S. Noda, Y. Sakamura, and S. Shimasaki, Phys. Rev. D 73, 096006 (2006).

[11] K. Agashe and R. Contino, Nucl. Phys. B742, 59 (2006); M. Carena, E. Ponton, J. Santiago, and C. E. M. Wagner, Nucl. Phys. B759, 202 (2006).

[12] W. D. Goldberger and M. B. Wise, Phys. Rev. Lett. 83, 4922 (1999); O. DeWolfe, D. Z. Freedman, S. S. Gubser, and A. Karch, Phys. Rev. D 62, 046008 (2000).

[13] B. Grzadkowski and J. Wudka, Phys. Rev. Lett. 97, 211602 (2006).

[14] L. Randall and R. Sundrum, Phys. Rev. Lett. 83, 3370 (1999).

[15] R. Contino and A. Pomarol, J. High Energy Phys. 11 (2004) 058

[16] A. Pomarol, Phys. Lett. B 486, 153 (2000).

[17] P. Horava and E. Witten, Nucl. Phys. B475, 94 (1996).

[18] A. Lukas, B. A. Ovrut, K. S. Stelle, and D. Waldram, Nucl. Phys. B552, 246 (1999).

[19] K. Freese, J. A. Frieman, and A. V. Olinto, Phys. Rev. Lett. 65, 3233 (1990); N. Arkani-Hamed, H.C. Cheng, P. Creminelli, and L. Randall, Phys. Rev. Lett. 90, 221302 (2003). 